\title{
Multi-model And Fuzzy PID Control for Fixed-wing UAV
}

\author{
Dali Zhou ${ }^{1, a^{*}}$, Qingbo Geng ${ }^{2, b}$ \\ ${ }^{1}$ School of Automation, Beijing Institute of Technology, Beijing, China \\ ${ }^{2}$ School of Automation, Beijing Institute of Technology, Beijing, China

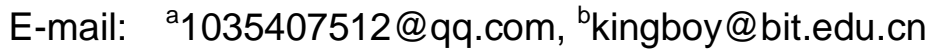

\begin{abstract}
Keywords: multi-model, adaptive control, fuzzy PID, F16
Abstract: This paper puts forward a kind of unmanned aerial vehicle (UAV) control method for longitudinal dynamics. Which includes velocity loop (inner loop) and height loop (outer loop). Multi-model adaptive control is used to control the velocity of UAV in the inner loop. The construction of model set and switching strategy of multi-model is introduced in this paper. Fuzzy PID is proposed to control the height of UAV in the outer loop. In this paper, we introduce the design of fuzzy rules, and use fuzzy controller to determine PID control parameters. Finally, the simulation of F16 model is carried out to verify that the improved control method is efficient and effective.
\end{abstract}

\section{Introduction}

In recent years, with applications of UAV expanding constantly in military and civilian areas, advantages of UAVs during tasks, such as convenience, safety and low cost, are more obvious. The UAV technology has become a hot topic, especially in civilian areas like remote sensing, mapping, traffic monitoring, geomagnetic detection and so on [1]. Additionally, the flight control system, as the core of UAV, determines the performance of UAV to a great extent. The PID controller is widely utilized in the traditional flight control system, but the inferior flight quality in terms of environmental adaptability make it difficult to get desirable flight quality. A variety of flight control methods have been developed for the application of UAV, such as nonlinear control techniques, which include feedback linearization, sliding mode control, robust control and so on [2].

The UAV system is of strong nonlinearity and time-varying characteristics which can be expressed as a set of complex nonlinear differential equations, thereby, in order to simplify the implementation and analysis of the closed-loop system, a simplified model of the longitudinal dynamics about F16 will be put forward. The multi-model adaptive control and fuzzy PID control scheme will be investigated and applied in this paper.

Multi-model adaptive controller mainly contains three parts [3]. Multi-model set, which is formed with multiple models according to uncertainty of parameters or structure of the plant. Controller set, which is constructed according to the multi-model set. Switching strategy, based on which corresponding controller is switched to the current controller.

With control error and differential of error as input of fuzzy PID controller, a set of fuzzy rules will be formulated according to control requirements to adjust PID control parameters. Specifically, the set of fuzzy rules is to figure out the relation between e, ec and PID control parameters, and the PID control parameters will be adjusted online through the developed fuzzy rules with respect to e and ec. Desirable dynamic and static performance will then be achieved [4].

The structure of the paper is organized as follows. The longitudinal model of F-16 is established in section 2. In section 3, the design of multi-model adaptive controller and the switching strategy are proposed. Fuzzy PID controller and fuzzy rules are introduced in section 4. Section 5 carries out 
a flight simulation and result analysis. Conclusions and further work are presented in section 6 .

\section{The Longitudinal Model of F16}

According to the principle of small perturbations, we can expand a nonlinear function to Taylor series and ignore the higher order terms to conclude the linear state space expressions. Thus, the UAV vertical movement at equilibrium point $\left(X_{\theta}, U_{\theta}\right)$ could carried out which is shown in [5].

$$
\dot{X}=F\left(X_{\varepsilon}, U_{\varepsilon}\right)+\frac{\partial F\left(X_{\varepsilon}, U_{\varepsilon}\right)}{\partial X}\left(X-X_{\theta}\right)+\frac{\partial F\left(X_{\varepsilon}, U_{\varepsilon}\right)}{\partial U}\left(U-U_{\varepsilon}\right)
$$

Let

$$
\dot{x}=\dot{X}-F\left(X_{\theta}, U_{\theta}\right)
$$

and

$$
x=X-X_{e}
$$

Then we have linearized state space equations

$$
\left\{\begin{array}{l}
\dot{x}=A x+B u \\
y=C x+D u
\end{array}\right.
$$

where

$$
x=\left[\begin{array}{llll}
\Delta V & \Delta \alpha & \Delta q
\end{array}\right]^{T}
$$

and

$$
u=\left[\Delta \delta_{T} \Delta \delta_{i}\right]^{T}
$$

where $\Delta V$ is the velocity of UAV, $\Delta \alpha$ is flight angle of attack, $\Delta \theta$ is pitch angle, $\Delta q$ is rate of pitch angle. $\Delta \delta_{T}$ is throttle opening which is used to control the velocity, $\Delta \delta_{g}$ is elevator angle that is utilized to control the flight height.

\section{Multi-Model Adaptive Control}

The inner loop is designed to control the velocity of a UAV. However, the model of a UAV will be changed with different heights and velocities. We can calculate multiple mathematical models of fixed-wing UAV, so multi-model adaptive control method is proposed.

As mentioned above, multi-model adaptive control contains three parts: the model set, the controller set and the switching strategy. And the global control scheme is shown in Fig.1.

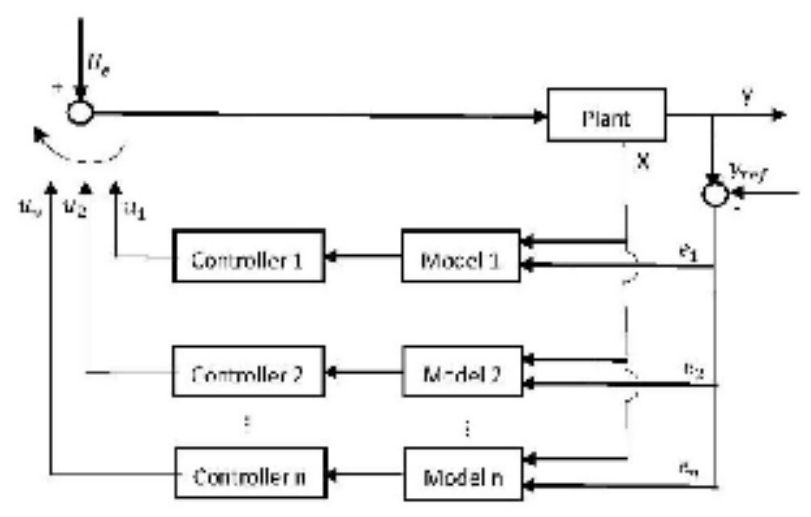

Fig. 1 multi-model adaptive control scheme

\section{A. The model set}

Various models are built based on different equilibrium points $\left(X_{\theta}, U_{\theta}\right)$, which are calculated by different velocities and altitudes, and $n$ models for inner loops and $m$ models for outer loops are thus built. 


\section{B. The controller set}

As shown in Fig.1, for reference model, we define $\bar{x}=\left[\begin{array}{ll}x & e\end{array}\right]^{T}$, then the augmented system can be obtained

$$
\bar{x}=\bar{A} \bar{x}+\bar{B} u
$$

where

$$
\bar{A}=\left[\begin{array}{ll}
A & 0 \\
C & 0
\end{array}\right], \overline{\mathrm{B}}=\left[\begin{array}{l}
B \\
0
\end{array}\right]
$$

Before the design of controllers, two assumptions should be given:

Assumption 1: For all models described by state space equations, $\left\{A_{i} B\right\}$ is controllable.

Assumption 2: For all models described by augmented state equations, $[\bar{A}, \bar{B}]$ is controllable.

The controllability mentioned in Assumption 1 and Assumption 2 ensures that the augmented system defined in (7) could be controllable, which means that the corresponding controller can be designed with state feedback method. So the linear quadratic optimal control method could be used in the inner loop.

From the block diagram of the velocity loop, as is shown in Fig.1, we can conclude the controller. In other words, $\mathrm{K}_{\mathrm{i}}(\mathrm{i}=1,2, \cdots, \mathrm{n})$ is a linear quadratic controller for its corresponding linearized model, so the control input of the linearized model is

$$
u_{i}=-K_{i} \bar{x}
$$

and $u$ is the control input of the longitudinal dynamics of F16,

$$
\overline{\mathrm{u}}=u_{i}+U_{\mathrm{e}}
$$

For linear system, its performance index is determined by quadratic function of the object state and control input, as the core part of linear quadratic optimal control. The object of linear quadratic optimal control method is based on state space expressions of linear system. Quadratic problem is to select control input to minimize the quadratic objective function on the condition of linear system constraints.

Performance index of the system is shown as follows:

$$
I=\frac{1}{2} \int_{0}^{\infty}\left(\bar{x}^{T} Q \bar{x}+\bar{u}^{T} R \bar{u}\right) d t
$$

where $Q$ and $R$ are constant matrices, and they meet the condition that $Q=Q^{T}, R=R^{T}$. Then the optimal feedback matrix is:

$$
K_{i}=R^{-1} \bar{B}^{T} P
$$

where $P$ is the solution of the algebraic Riccati equation.

\section{Switching strategy}

$$
P \bar{A}+\bar{A}^{T} P-P \bar{B} R^{-1} \bar{B}^{T} P+Q=0
$$

In the process of unmanned aerial vehicle (UAV) flight, the aircraft model will change with the flight velocity and height. If $n$ models are selected, then we will obtain $n$ equilibrium points in order to construct $n$ reference models. The equilibrium points are solved with state variables in (6). And we divide velocity into $n$ parts from $V_{\min }$ to $V_{\max }$ as follows.

$$
\begin{aligned}
V_{i} & =\sqrt{\frac{i-1}{n-1}}\left(V_{\max }-V_{\min }\right)+V_{\min } \\
i & =1,2 \ldots, n
\end{aligned}
$$

If the velocity of UAV closes to $V_{i}$, then the $i$ th model will be chosen as current controller. We can divide flight height into $m$ parts from $H_{\min }$ to $H_{\max }$ in the same way.

Where 


$$
\begin{aligned}
& H_{i}=\frac{i-1}{m-1}\left(H_{\max }-H_{\min }\right)+H_{\min } \\
& i=1,2 \ldots, m
\end{aligned}
$$

So, there are $m * n$ models overall. The multi-model adaptive controller will change with velocity and height automatically.

\section{Fuzzy PID Controller Design}

The outer loop is used to control the altitude of the UAV, whose diagram of altitude loop is shown in Fig.2.

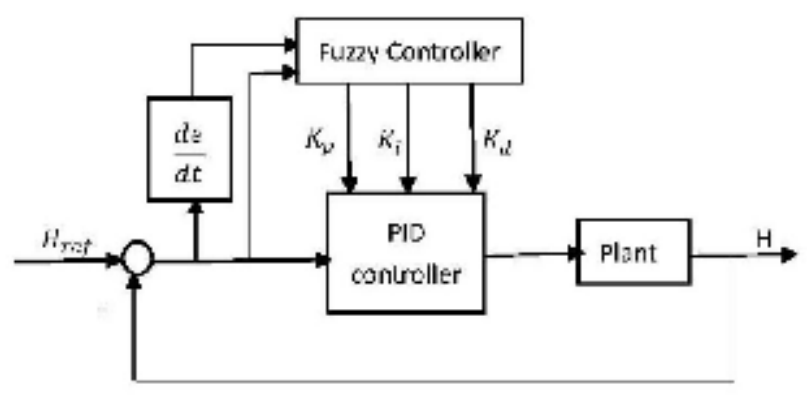

Fig. 2 Fuzzy PID controller method

Fuzzy PID controller is utilized to control the altitude of UAV. The effect of fuzzy PID parameters self-tuning is to find the fuzzy relation between PID's three parameters and error $e_{H}(e)$, error rate $e_{\mathrm{H}}^{*}(\mathrm{ec})$. Then detect $\mathrm{e}$ and ec constantly in operation, and then modify PID's three parameters online based on fuzzy control theory to acquire good dynamic and static characteristics for control object. When e is bigger, smaller $K_{p}$ is applied in order to avoid supersaturated, and when e is smaller, bigger $K_{p}$ should be used [6]. Then the fuzzy rules are shown from Table.1 to Table.3. Here we choose 25 fuzzy rules in each PID parameters. The range of input variables e and ec is $\{-2,-1,0,1,2\}$. Where the quantization factor of $\mathrm{K}_{\bullet}$ is 0.2 , and the quantization factor of $\mathrm{K}_{\oplus e}$ is 200 . The scale factors of $K_{p}{ }^{\prime}, K_{d}{ }^{\prime}, \alpha$ are designed separated as 1.75, 0.1, 1.25 .

Table 1. Fuzzy rules of $\boldsymbol{K}_{p}{ }^{\prime}$

\begin{tabular}{|c|c|c|c|c|c|}
\hline$K_{p}{ }_{e}$ & $\mathrm{NB}$ & $\mathrm{NS}$ & $\mathrm{ZO}$ & $\mathrm{PS}$ & $\mathrm{PB}$ \\
\hline $\mathrm{NB}$ & $\mathrm{B}$ & $\mathrm{B}$ & $\mathrm{B}$ & $\mathrm{B}$ & $\mathrm{B}$ \\
\hline $\mathrm{NS}$ & $\mathrm{S}$ & $\mathrm{B}$ & $\mathrm{B}$ & $\mathrm{B}$ & $\mathrm{S}$ \\
\hline ZO & S & S & B & S & S \\
\hline PS & S & B & B & B & S \\
\hline PB & B & B & B & B & S \\
\hline
\end{tabular}

Table 2. Fuzzy rules of $K_{d}{ }^{\prime}$

\begin{tabular}{|c|c|c|c|c|c|}
\hline$K_{d}{ }^{d e c}$ & NB & NS & ZO & PS & PB \\
\hline NB & S & S & S & S & S \\
\hline NS & B & B & S & B & B \\
\hline ZO & B & B & B & B & B \\
\hline PS & B & B & S & B & B \\
\hline PB & S & S & S & S & S \\
\hline
\end{tabular}

Table 3. Fuzzy rules of $\alpha$

\begin{tabular}{|c|c|c|c|c|c|}
\hline e & NB & NS & ZO & PS & PB \\
\hline NB & S & S & S & S & S \\
\hline NS & MS & MS & S & MS & M \\
\hline
\end{tabular}




\begin{tabular}{|c|c|c|c|c|c|}
\hline ZO & B & MS & MS & MS & B \\
\hline PS & MS & MS & S & MS & M \\
\hline PB & S & S & S & S & S \\
\hline
\end{tabular}

The fuzzy controller is a two input, three output system, and the fuzzy membership function for input variables $e_{H}, e_{H}^{*}$ and output variable $\alpha$ is triangular function. The fuzzy membership function for output variables $\mathrm{K}_{d}{ }^{\prime}$ and $\mathrm{K}_{p}{ }^{\prime}$ is Gaussian function.

The PID parameters are concluded as follows:

$$
\begin{aligned}
& K_{p}=\left(K_{p, \text { max }}-K_{p, \text { min }}\right) K_{p}^{\prime}+K_{p, \text { min }} \\
& K_{d i}=\left(K_{d, \max }-K_{d, \text { min }}\right) K_{d}^{\prime}+K_{d, \text { min }} \\
& K_{i}=K_{p}^{\prime 2} /\left(\alpha K_{d}\right)
\end{aligned}
$$

and PID controller equation is

$$
\theta_{r e f}=K_{p}\left(e_{H}+K_{i} \int e_{H} d t+K_{d} \frac{d \theta_{H}}{d t}\right)
$$

\section{Simulation}
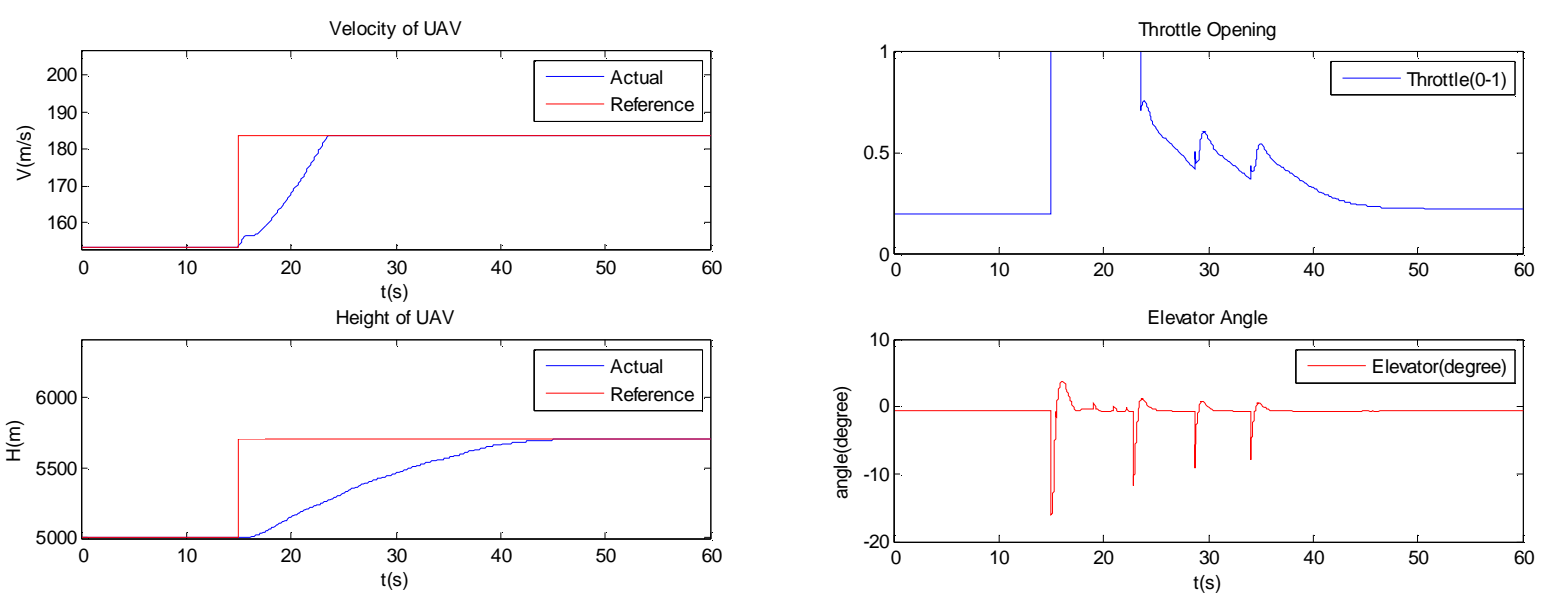

(a) Step response of velocity and height

(b) Control input
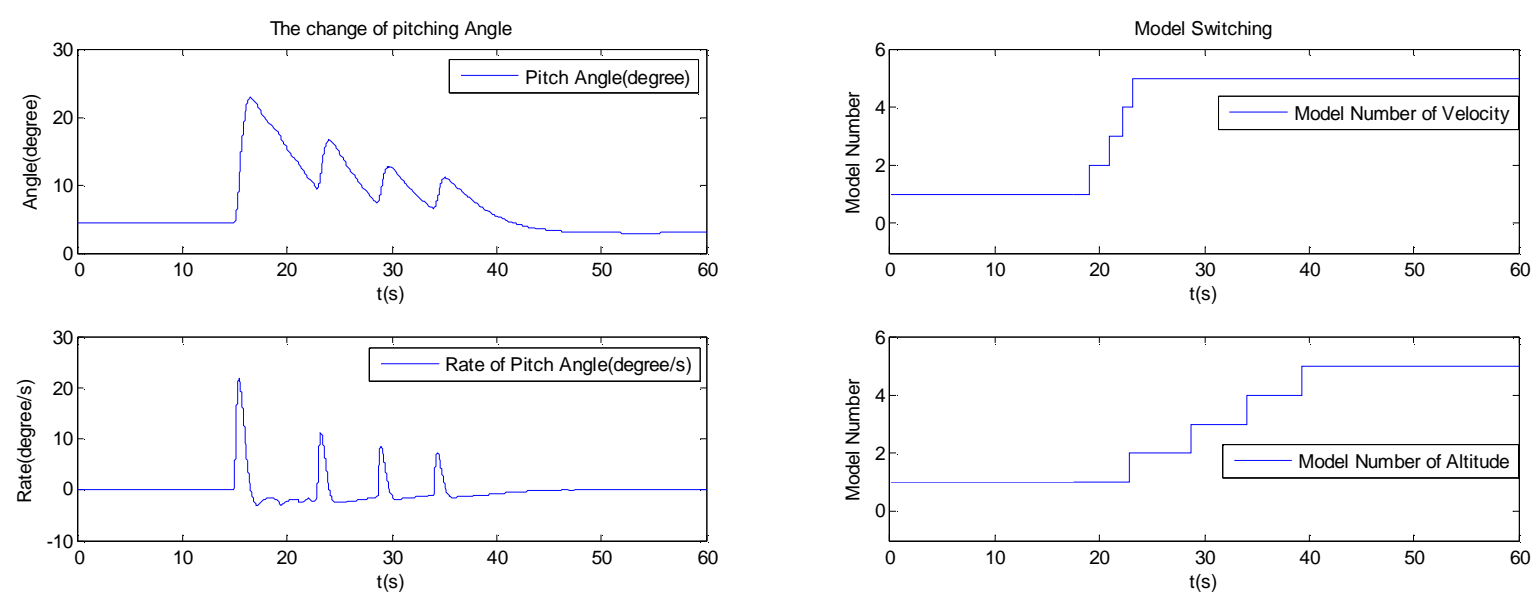

(c) Pitch angle and the rate

(d) Model switching

Fig 3. The simulation results 
From Fig.3 (a), it can be seen that the output can track the reference without static error. The adjustment of control input in the process of flight is shown in Fig.3 (b), and eventually it tends to stable. Fig.3 (c) presents the dynamic change of pitch angle and the rate. The model switching is displayed in Fig.3 (d).

\section{Conclusion}

The main contribution of this paper is to study the method of multi-model adaptive control and fuzzy PID in the use of UAV. Model selection and switching strategy have been proposed in the paper. The multi-model controller will change its reference model according to the actual environment. In order to improve the performance of PID controller in the outer loop, we introduce fuzzy controller to adjust PID control parameters online. Finally, F16 model has been used to verify the feasibility of the scheme.

\section{References}

[1] Hongxi Yang, Qingbo Geng, The Design of Flight Control System for Small UAV with Static Stability, Proceedings of 2011 2nd International Conference on Mechanic Automation and Control Engineering, p799-803, 2011.

[2] Yeh Fu-Kuang, Huang Chia-Wei, Huang Jian-Ji, Adaptive fuzzy sliding-mode control for a mini-UAV with propellers, Proceedings of the SICE Annual Conference, p645-650, 2011.

[3] Wei Wang, Xiaoli Li, Multi-model adaptive Control, Science Press, Beijing, China, 2001.

[4] Ding Wen-shuang, Hu Yu-wen, Lu Wen-qi, Liang Jiao-yan, Fuzzy PID Parameter Self-Turning Control for PMSM Servo System, Small\&Special Electrical Machines, 39(5), p17-21, 2011.

[5] Brian L. Stevens, Frank L. Lewis, Aircraft Control and Simulation, Wiley-Interscience, New York, U.S.A, 1992.

[6] Dingxue Zhang, Zhen Xu, Fuzzy PID control in the process control, Proceedings of the 2012 International Symposium on Instrumentation, p587-91, 2012. 